\title{
Analytical correlation correction of the chemical potential of solid solutions
}

\author{
A. Udyansky, ${ }^{1}$ V. N. Bugaev, ${ }^{1}$ W. Schweika, ${ }^{2}$ O. Shchyglo, ${ }^{1}$ H. Reichert, ${ }^{1}$ and H. Dosch ${ }^{1,3}$ \\ ${ }^{1}$ Max-Planck-Institut für Metallforschung, Heisenbergstrasse 3, D-70569 Stuttgart, Germany \\ ${ }^{2}$ Institut für Festkörperforschung, Forschungszentrum Jülich, D-52425 Jülich, Germany \\ ${ }^{3}$ Institut für Theoretische und Angewandte Physik, Universität Stuttgart, D-70569 Stuttgart, Germany
}

(Received 16 January 2005; published 28 April 2005)

\begin{abstract}
We derive an analytical expression for the chemical potential of disordered binary alloys using a reciprocal space approach. The main characteristic of the formalism is that it does not limit the effective radius of atomic interaction and correlations in the system. The lattice displacements caused by atomic size mismatch can be naturally introduced into this formalism. A comparison with results from Monte Carlo simulations shows very good agreement. The new analytical expression for the chemical potential can be widely applied, e.g., for the calculation of phase diagrams as well as surface segregation profiles in nanoconfined systems.
\end{abstract}

DOI: 10.1103/PhysRevB.71.140201

PACS number(s): 64.60.Cn, 61.66.Dk, 05.50.+q

The chemical potential of a solid solution $A-B, \mu=\mu_{A}$ $-\mu_{B}$, where $\mu_{A}$ and $\mu_{B}$ are the chemical potentials of the alloy components, is a fundamental thermodynamical quantity, since, at thermodynamic equilibrium, the chemical potentials of all the parts of the alloy are equal. This condition is applied in many areas of solid state physics and materials science, including the study of phase diagrams, spinodal decomposition or surface segregation phenomena. From the condition that the chemical potentials in all product phases must be equal in systems undergoing spinodal decomposition, the concentration in each of them can be found. ${ }^{1-4}$ In surface segregation theory, the condition that the chemical potential is equal in all near-surface layers and in the bulk part of the alloy provides a set of equations for the atomic concentration in every layer. ${ }^{5-7}$

For the calculation of the chemical potential of solid solutions Monte Carlo (MC) simulations ${ }^{8}$ or analytical statistical-thermodynamic approaches ${ }^{2,3,6}$ can be used. The MC method is characterized by a high accuracy, because it avoids additional simplifying assumptions, and can be used as a standard in statistical thermodynamics. On the other hand, simple models and analytical expressions for thermodynamic quantities are extremely important, since they not only elucidate qualitative trends but also allow us to express one physical property of the system via others. In addition, analytical approaches demand in general much less computational efforts in comparison to MC simulations.

Among the analytical statistical-thermodynamic approaches for the calculation of the chemical potential, the single-site mean-field approximation (MFA) and several versions of the cluster-variation method (CVM) are commonly used. The MFA ignores correlation effects in the mutual arrangement of the different alloy components. ${ }^{3,9}$ The CVM, which takes correlations into account, ${ }^{2,4,9}$ involves cumbersome analytic equations and a nonlinear increase in calculation time with increasing radius of atomic interaction.

Real systems, such as metallic alloys, are often characterized by a large radius of atomic interaction. One source of long-ranging interactions is atomic size mismatch, which is generally present in alloy systems. It results in an infinitely ranging strain-induced interaction between atoms. ${ }^{2,3,10} \mathrm{An}-$ other type of long-ranging interaction is found in alloys with flat portions in the Fermi surface causing Fermi surface nesting effects. ${ }^{11}$ The reciprocal space modification of the singlesite MFA does not suffer from the problems mentioned above, although it is less accurate in the vicinity of a phase transition, and, moreover, might give qualitatively wrong results in the case of frustrated lattices. 3,9

The aim of this paper is to derive a simple and accurate analytical expression for the chemical potential of a disordered alloy, which takes correlation effects into account. For the derivation of the free energy of the alloy we use a reciprocal space ( $k$-space) approach based on an iterative correlation correction procedure. Instead of performing the calculations within a thermodynamic perturbation theory ${ }^{10}$ we integrate the configurational free energy with respect to the inverse temperature. ${ }^{8,9}$

For the calculation of the chemical potential we start with the free energy of the system (see, e.g., Ref. 11)

$$
\mu=\frac{1}{N} \frac{\partial F}{\partial c},
$$

where $F$ is the configurational free energy, $c$ the concentration of the component $A$, and $N$ the number of lattice sites in the alloy crystal. In MC simulations the numerical value of the configurational free energy of the alloy is then calculated using the exact relation ${ }^{8,9}$

$$
F=k_{B} T \int_{0}^{1 / k_{B} T} E\left(T^{\prime}\right) d\left(1 / k_{B} T^{\prime}\right)-T S_{0},
$$

where $k_{B}$ is the Boltzmann constant, $T$ the temperature, $E$ the internal configurational energy of the alloy, and $S_{0}$ the value of the configurational entropy, calculated in the hightemperature limit.

In the following we split the internal configurational energy of the alloy into a sum of two terms

$$
E=E_{0}+\Delta E,
$$

where $E_{0}$ denotes the internal configurational energy in the high-temperature limit where the correlations in the occupation of lattice sites are vanishing. $\Delta E$ is the energy contribu- 
tion caused by atomic correlations. Equation (2) can then be written as

$$
F=F_{0}+k_{B} T \int_{0}^{1 / k_{B} T} \Delta E\left(T^{\prime}\right) d\left(1 / k_{B} T^{\prime}\right),
$$

where $F_{0}=E_{0}-T S_{0}$.

It is important to note, that the mean-field (MF) expression for the configurational energy and entropy of an alloy are asymptotically exact in the high-temperature limit. ${ }^{12}$ Therefore, it can be used for the calculation of $F_{0}$. In addition, in the single-site MFA for the disordered state of the alloy, the values $S_{0}$ and $E_{0}$ do not depend on temperature.

Assuming the atomic interactions to be pairwise, the energy contribution caused by atomic correlations is given by

$$
\Delta E=\frac{1}{2 N} \sum_{\vec{k}} V_{\vec{k}} \varepsilon_{\vec{k}}
$$

where $V_{\vec{k}}$ and $\varepsilon_{\vec{k}}$ are the Fourier transforms of the two-body mixing potential and the correlation function, respectively. ${ }^{13}$ The summation in Eq. (5) is carried out over all wave vectors in the first Brillouin zone. In the following, the definition $f_{\vec{k}}=\sum f_{\vec{R}} \mathrm{e}^{-i \vec{k} R}$ for the Fourier transform is used for any function $f_{R}$ of the real space vector $\vec{R}$ where the summation is carried out over all sites of the alloy crystal.

Substituting Eq. (5) in Eq. (4) gives

$$
F=F_{0}+\frac{k_{B} T}{2 N} \sum_{\vec{k}} V_{\vec{k}} \int_{0}^{1 / k_{B} T} \varepsilon_{\vec{k}}\left(T^{\prime}\right) d\left(1 / k_{B} T^{\prime}\right),
$$

where we assume that the interaction potentials $V_{\vec{k}}$ do not depend on temperature and concentration.

In the presence of atomic size mismatch, the mixing potential can be represented as $V_{\vec{k}}=V_{\vec{k}}^{\mathrm{ch}}+V_{\vec{k}}^{s-i}$, i.e., as a sum of a chemical part determined for the undistorted (perfect) lattice and an effective strain-induced part. ${ }^{2,3,14}$

The correlation function $\varepsilon_{\vec{k}}=\varepsilon_{\vec{k}}(T)$ can be calculated iteratively using various $k$-space statistic-thermodynamical approximations starting from the MFA. In the following we use the Krivoglaz-Clapp-Moss (KCM), ${ }^{11}$ the spherical model $(\mathrm{SM}),{ }^{13}$ and the recently developed ring $^{10}$ approximation (RA). These approximations are listed in the order of increasing accuracy in the correlation function. ${ }^{10}$

Figure 1 displays the iterative procedure for the calculation of the correlation function and the free energy. In each loop the chemical potential of the system can be calculated with increasing accuracy starting with the MFA value for the chemical potential

$$
\mu_{\mathrm{MFA}}=\Phi+V_{\vec{k}=0} c+k_{B} T \ln \frac{c}{1-c},
$$

with $\Phi$ as the energy of injection of an $A$ sort atom into a crystal lattice site (the unary mixing potential). ${ }^{15}$

In the first loop of this iterative procedure, correlations within the SM approximation are taken into account. Within this approximation ${ }^{10,13}$ the correlation function is given by

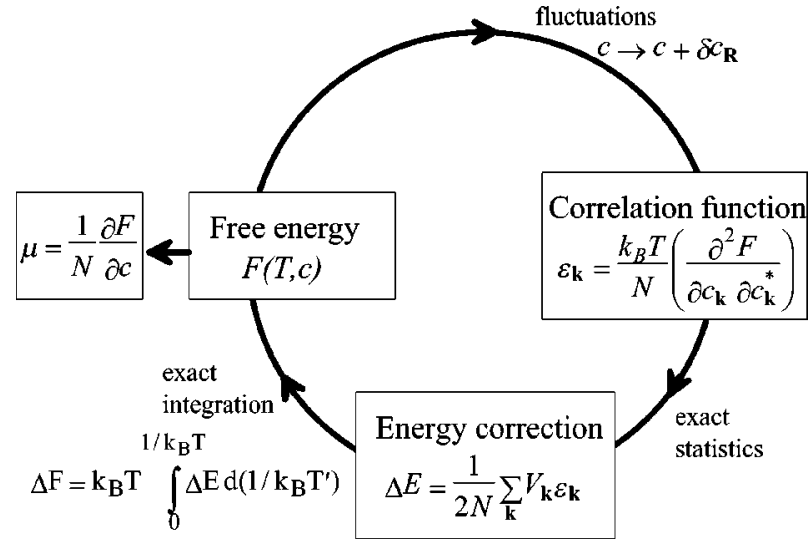

FIG. 1. Iterative procedure for the calculation of the correlation corrections to the free energy and the chemical potential.

$$
\varepsilon_{\vec{k}}^{\mathrm{SM}}=\frac{c(1-c)}{1+c(1-c)\left(V_{\vec{k}}+\lambda\right) / k_{B} T},
$$

with $\lambda$ as a Lagrangian multiplier, constrained by the condition

$$
\frac{\partial F}{\partial \lambda}=0
$$

Note that the case $\lambda=0$ in Eq. (8) corresponds to the KCM equation.

Substituting Eq. (8) into Eq. (6) and using Eq. (9), we obtain the normalization condition

$$
N^{-1} \sum_{\vec{k}} \varepsilon_{\vec{k}}^{\mathrm{SM}}=c(1-c) .
$$

By using Eq. (6) and the definition in Eq. (1) one can arrive again at an analytical expression for the chemical potential of the alloy

$$
\mu_{\mathrm{SM}}=\mu_{\mathrm{MFA}}+\frac{1-2 c}{2 N c(1-c)} \sum_{\vec{k}} V_{\vec{k}} \varepsilon_{\vec{k}}^{\mathrm{SM}}
$$

This is the key result of this study.

In order to test the numerical accuracy of Eq. (11) we have performed extensive MC simulations and compared the results with calculations in the various approximations. The MC simulations were done in the grand canonical ensemble. The standard Metropolis single spin-flip MC method was used. Typical model crystals with periodic boundaries varied in size from $10 \times 10 \times 10$ to $60 \times 60 \times 60$ unit cells to control possible finite size effects. The chemical potential was adjusted in the simulations in order to achieve the desired composition of the alloy. The MC results are shown in Figs. 2-4 as bold blue lines and serve as an exact reference for the analytical Eqs. (7) and (11).

First we present results for a series of model systems. For the first two systems two different interaction models (a) $V_{1}>0, V_{S}=0, s \geqslant 2$, and (b) $V_{1}>0, V_{2}=-0.5 V_{1}, V_{S}=0, s$ $\geqslant 3$ have been used with a fixed concentration of $A$-atoms $c_{A}=0.1$. In order to test the numerical results for a different 


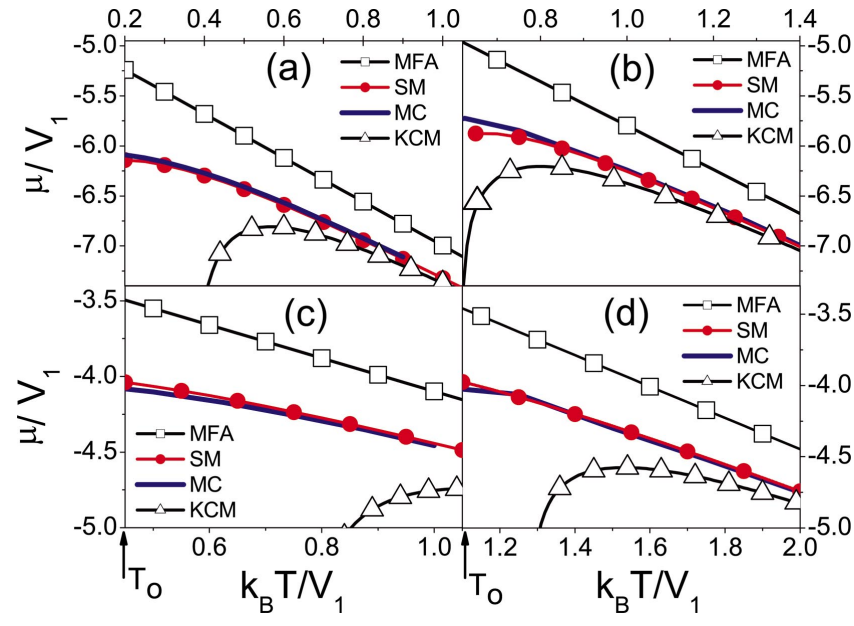

FIG. 2. (Color) Dependence of the reduced chemical potential on the reduced temperature, obtained by MC simulations, the MFA, the KCM, and the SM approximation for $c_{A}=0.1$ and (a) $V_{1}>0$, $V_{s}=0(s \geqslant 2)$, (b) $V_{1}>0, V_{2}=-0.5 V_{1}, V_{s}=0(s \geqslant 3)$, and for $c_{A}$ $=0.25$ and (c) $V_{1}>0, V_{s}=0(s \geqslant 2)$, (d) $V_{1}>0, V_{2}=-0.5 V_{1}, V_{s}$ $=0(s \geqslant 3)$.

concentration we present in a second series of calculations and simulations with the same interaction models for a concentration of $A$-atoms $c_{A}=0.25$.

In Fig. 2 the results for $\mu_{\mathrm{MFA}}, \mu_{\mathrm{KCM}}$, and $\mu_{\mathrm{SM}}$ are shown for the temperature range $T>T_{0}$, where $T_{0}$ denotes the orderdisorder transition temperature. ${ }^{16}$ This demonstrates that the MFA systematically overestimates the chemical potential (upper bound), while the KCM approximation systematically underestimates the chemical potential of the system (lower bound).

Figure 2 also shows that the SM approximation provides a high numerical accuracy, since the results of calculations using MC simulations and the SM approximation practically coincide with each other in a wide temperature range, except the narrow interval near the phase transition temperature, where the maximum deviation is less than $1 \%$. The results obtained within the single-site MFA (which neglects correla-

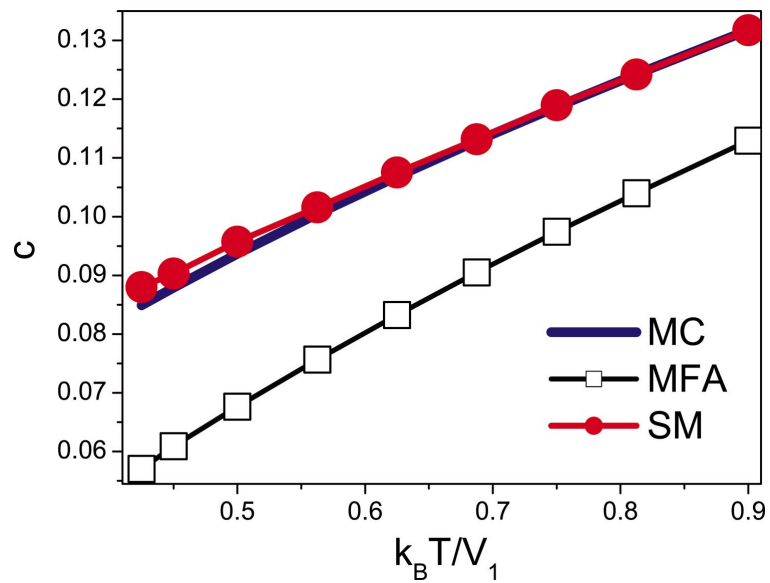

FIG. 3. (Color) Temperature dependence of the concentration calculated within the SM approximation for a model system described by a fixed chemical potential $\mu / V_{1}=-7$.

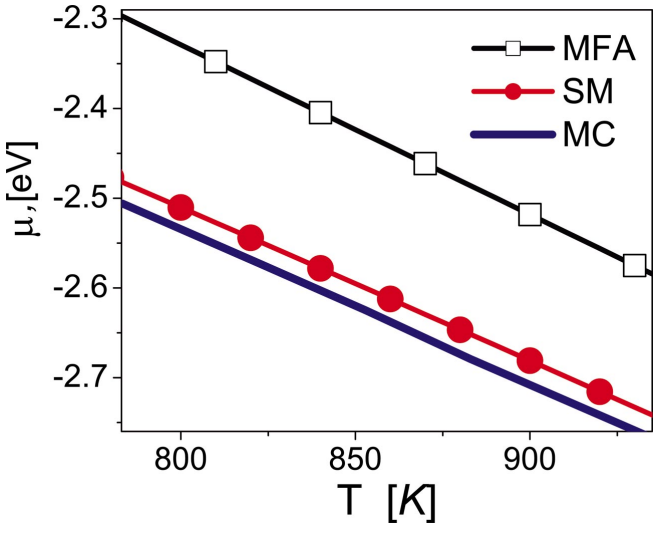

FIG. 4. (Color) Temperature dependence of the chemical potential for the alloy $\mathrm{Ni}_{0.89} \mathrm{Cr}_{0.11}$, obtained with $\mathrm{MC}$ simulations, within the MF approximation, and within the SM approximation. The effective atomic interaction potential in 30 shells is taken from Ref. 17.

tion effects in the atomic distribution) display deviations to the exact values of more than $10 \%$ in all cases considered here. The KCM approximation approaches the MC results at high temperatures and gives the biggest discrepancy from the MC results in the interval close to the phase-transition temperature. Although this discrepancy is decreasing when interactions in the second coordination shell are taken into account, this approximation gives nonadequate trends in close vicinity of the transition temperature.

In Fig. 3 we demonstrate that the formalism developed above can be readily applied for the determination of the concentration as function of temperature at a fixed chemical potential. The results of the MC simulations and the calculations done in the SM approximation are very close within a wide range of temperature. Notice that the MF calculations exhibit systematic errors up to $30 \%$.

Figure 4 shows that the tendencies demonstrated in the case of the short-range interactions (Fig. 2) are also found for the real alloy $\mathrm{Ni}_{0.89} \mathrm{Cr}_{0.11}$ in the temperature range under consideration. In this case the effective interaction is ranging up the 30th neighbor shell. ${ }^{17}$ The chemical potentials calculated within the ring approximation using Eq. (12) and calculated within the spherical model using Eq. (8) are identical within $0.3 \%$. The systematic deviation between the MC and the SM results is approximately $1 \%$, and between the $\mathrm{MF}$ and the MC approximately $10 \%$.

The numerical tests of the correlation correction procedure performed in this paper using the spherical model for the correlation function demonstrate that the comparatively simple analytical Eq. (11) derived for the chemical potential of a binary alloy has a high numerical accuracy in a wide temperature range, both in the case of model systems with short-range atomic interactions, and in the case of a real alloy with long-range interactions.

In those cases, where even higher accuracies for the determination of the chemical potential are required, a numerical solution of Eqs. (1) and (6) can be performed employing the correlation function calculated in the second loop of the iterative procedure (see Fig. 1) which results in the RA. Within the RA the correlation function reads 


$$
\varepsilon_{\vec{k}}^{\mathrm{RA}}=\frac{c(1-c)}{I+c(1-c) V_{\vec{k}}^{\mathrm{eff}} / k_{B} T},
$$

with

$$
I=N^{-1} \sum_{\vec{k}}\left[1+c(1-c)\left(V_{\vec{k}}+\lambda\right) / k_{B} T\right]^{-1},
$$

and

$$
V_{\vec{k}}^{\mathrm{eff}}=V_{\vec{k}}-\frac{(1-2 c)^{2}}{2 N k_{B} T c^{2}(1-c)^{2}} \sum_{\vec{q}}\left(V_{\vec{q}}+\lambda\right)\left(V_{\vec{k}-\vec{q}}+\lambda\right) \varepsilon_{\vec{q}}^{\mathrm{SM}} \varepsilon_{\vec{k}-\vec{q}}^{\mathrm{SM}} .
$$

The Lagrangian multiplier $\lambda$ in Eq. (14) should be calculated using the normalization condition given by Eq. (10). We emphasize here that, in contrast to the corresponding values in the KCM and SM approximation, the values for $I$ and $V_{\vec{k}}^{\text {eff }}$ are temperature dependent in the RA. Therefore, the integration over the inverse temperature in Eq. (6) and the calculation of the derivative with respect to concentration in Eq. (1) can be performed only numerically and in turn the equation for the chemical potential can no longer be written in a closed analytical form. The chemical potential determined within the RA is virtually identical to the SM results and is, therefore, not shown in the figures.

In conclusion we emphasize, that, due to the use of a $k$-space approach, the radius of the effective interaction has no limitation; thus the presented formalism naturally takes into account all long-ranging strain-induced effects. ${ }^{2,3,11}$ It can furthermore be generalized to systems governed by many-body interactions ${ }^{14,18}$ and by magnetic interactions. ${ }^{9,19}$ The derivation of the analytical expressions for the chemical potential has been carried out independently from the dimension of the system under consideration.

Therefore, this formalism can be applied to material science problems of current interest, in particular to calculate phase diagrams of "real" binary alloys, to study complex multicomponent, amorphous, and fluid systems, and to investigate ordering and segregation phenomena at surfaces, interfaces, and in thin films.
${ }^{1}$ R. Kikuchi and D. de Fontaine, Scr. Metall. 10, 995 (1976).

${ }^{2}$ D. de Fontaine, Solid State Phys. 34, 73 (1979).

${ }^{3}$ A. G. Khachaturyan, Theory of Structural Transformations in Solids (Wiley, New York, 1983).

${ }^{4}$ V. G. Vaks, Phys. Rep. 391, 157 (2004).

${ }^{5}$ F. L. Williams and D. Nason, Surf. Sci. 45, 377 (1974).

${ }^{6}$ G. Kerker, J. L. Morán-López, and K. H. Bennemann, Phys. Rev. B 15, 638 (1977).

${ }^{7}$ M. Polak and L. Rubinovich, Surf. Sci. Rep. 38, 127 (2002).

${ }^{8} \mathrm{~K}$. Binder, in Monte Carlo Methods in Statistical Physics, edited by K. Binder (Springer, Berlin, 1979).

${ }^{9}$ F. Ducastelle, Order and Phase Stability in Alloys (NorthHolland, Amsterdam, 1991).

${ }^{10}$ V. N. Bugaev and R. V. Chepulskii, J. Phys.: Condens. Matter 10, 7309 (1998).
${ }^{11}$ M. A. Krivoglaz, Diffuse Scattering of X-Ray and Neutron by Fluctuations (Springer, Berlin, 1996).

${ }^{12}$ R. A. Suris, Fiz. Tverd. Tela (Leningrad) 4, 1154 (1962) [Sov. Phys. Solid State 4, 850 (1962)].

${ }^{13}$ R. Brout, Phase Transitions (Benjamin, New York, 1965).

${ }^{14}$ V. N. Bugaev et al., Phys. Rev. B 65, 180203(R) (2002).

${ }^{15}$ V. N. Bugaev and R. V. Chepulskii, Acta Crystallogr. 51, 456 (1995).

${ }^{16}$ R. V. Chepulskii and V. N. Bugaev, Solid State Commun. 105, 615 (1998).

${ }^{17}$ W. Schweika and H.-G. Haubold, Phys. Rev. B 37, 9240 (1988).

${ }^{18}$ R. V. Chepulski, J. Phys.: Condens. Matter 11, 8645 (1999).

${ }^{19}$ M. F. R. Drautz and J. M. Sanchez, J. Phys.: Condens. Matter 16, 3843 (2004). 